\title{
A influência do método pilates na musculatura do assoalho pélvico em mulheres no climatério: estudo de caso
}

\author{
The method pilates effects on pelvic floor of climacteric women: \\ a case of study
}

Josiane Teresinha Bertoldi, ${ }^{1}$ Ariane Maiara Medeiros, ${ }^{1}$ Sabrina Oliveira Goulart ${ }^{1}$

${ }^{1}$ Associação Catarinense de Ensino - Faculdade Guilherme Guimbala (FGG/ACE), Joinvile, SC, Brasil.

Recebido em: Setembro 2015 / Aceito em: Dezembro 2015

bertoldi.josi@gmail.com

\section{RESUMO}

Objetivo: avaliar a influência dos exercícios do método Pilates na força de contração da musculatura do assoalho pélvico (MAP) e na qualidade de vida (OV) de mulheres no climatério. Método: amostra composta por duas mulheres (1) e (2), com 50 e 48 anos respectivamente, sem disfunção da MAP. A pré e pós-avaliação foi composta pelos protocolos: avaliação visual funcional (AFA), teste dos cones vaginais (TCV), teste de força com perineômetro modelo perina, quark ${ }^{\circledR}$ para fibras rápidas (TPFR) e para fibras lentas (TPFL), e avaliação da QV com o Women's Health Questionnaire (WHQ). O tratamento consistiu em 10 sessões de 50 minutos, 03 vezes por semana, compostas por exercícios selecionados do método Pilates. Resultados: AFA e TCV o grau de força foi máximo no pré e pós-teste, entretanto notou-se melhora da contração isolada da MAP; no TPFR houve incremento de força de $45,83 \%$ (1) e $362,50 \%$ (2), no TPFL o ganho foi de $82,43 \%$ (1) e $209,38 \%$ (2); no WHQ houve variação positiva nas dimensões: sintomas somáticos, vasomotores, menstruais, atratividade e depressão. Considerações finais: o método Pilates promove aumento da força de contração da MAP, com discreta influência na autopercepção da QV de mulheres no período do climatério, sem patologias associadas.

Palavras-chave: Diafragma Pélvico; Climatério; Saúde da Mulher; Fisioterapia.

\section{ABSTRACT}

Objective: to evaluate the influence of Pilates exercises on the force of contraction of the pelvic floor muscles (PFM) and quality of life (OOL) of women during menopause. Method: two women (1 and 2), 50 and 48 years respectively, with no impairment of PFM. The pre- and post-assessment was made by the protocols: Female PFM Function and Strength (FS), Test of vaginal cones (TVC), Strength Assessment with perineometer model Perina, Quark ${ }^{\circledR}$ for Fast-twitch (APFT) and Slowtwitch (APST), and QoL assessment with the Women's Health Questionnaire (WHQ). Treatment consisted of 10 sessions of 50 minutes, 03 times a week, composed of selected exercises of Pilates method. Results: FS and TCV, the degree of force was most pre and post-test, however noticed an improvement isolated contraction of MAP; APFT was increased by $45.83 \%$ (1) and 362.50\% (2); APST gain was $82.43 \%$ (1) and $209.38 \%$ (2); WHO there was a positive variation in the dimensions: somatic symptoms, vasomotor, menstrual, attractiveness and depression. Closing remarks: the Pilates method promotes increase in PFM contraction force, with slight influence on $Q O L$ of women in the climacteric period without pathologies.

Keywords: Pelvic Floor Muscle; Climacteric; Women's Health; Physical Therapy. 


\section{INTRODUÇÃO}

O assoalho pélvico (AP) representa o conjunto das partes moles que fecham a pelve suportando o peso das vísceras em ortostase e é formado por todas as estruturas que estão contidas entre o peritônio e a pele da vulva. A região é constituída por músculos, fáscias e ligamentos que garantem o suporte dos órgãos abdominais e pélvicos, controlam a continência urinária e fecal, contrabalançam os efeitos da pressão intra-abdominal, além da sua participação na função sexual e na passagem do feto no parto. ${ }^{1-3} \mathrm{~A}$ musculatura do assoalho pélvico (MAP) é composta pelo diafragma pélvico e urogenital, sendo o diafragma pélvico composto pelos músculos coccígeo e elevadores do ânus, e o diafragma urogenital pelos músculos bulbocavernoso, transverso superficial do períneo e isquiocavernoso. ${ }^{4}$

A anatomia do grupo muscular denominado de elevador do ânus, exposta por Standring, apud Ribeiro, ${ }^{5}$ define que este é o maior componente do assoalho pélvico, dividido em duas partes, sendo a primeira representada pelos músculos ileococcígeos bilaterais, que formam uma plataforma contínua e se estendem do osso púbis à espinha isquiática. A segunda parte do elevador do ânus é o chamado músculo pubovisceral, que inclui os músculos puborretal e pubococcígeo. Este último tem forma de $U$, circundando o hiato urogenital. ${ }^{5} \mathrm{O}$ estrogênio age sobre o tônus e o trofismo desta musculatura, além de influenciar no trofismo da mucosa uretral e vaginal, no tecido conjuntivo dos tratos urinários e genital e sobre a vascularização periuretral. ${ }^{6}$

Os sistemas urinário e reprodutivo têm a mesma origem embriológica e apresentam-se ligados anatômica e funcionalmente. Ambos os sistemas respondem à ação dos esteróides sexuais ovarianos para funcionarem apropriadamente. Por isso o hipoestrogenismo tem potencial para provocar efeitos diversos sobre o trato urogenital. ${ }^{6,7}$

O déficit hormonal torna-se evidente durante o período do climatério, um marco biológico que representa a etapa de transição entre o período reprodutivo e não-reprodutivo, que ocorre aproximadamente entre 40 e 55 anos. ${ }^{8-10}$ Neste período ocorre o esgotamento dos folículos ovarianos e segue-se a queda progressiva da secreção de estrogênio, culminando com a interrupção definitiva dos ciclos menstruais (menopausa) e o surgimento de sintomas característicos. ${ }^{8-10} \mathrm{Na}$ maioria das mulheres, o climatério desencadeia sintomas vasomotores, psicológicos, urogenitais, sexuais e de distúrbios do sono, derivados do declínio de estrogênio. ${ }^{11}$

É aceito que o relaxamento da musculatura do assoalho pélvico, consequente à perda de força muscular perineal, é comum em mulheres e tendem a aumentar com a idade..$^{12,13}$ Os baixos níveis de estrogênio associados à menopausa têm sido considerados como o fator causal de hipotrofia do assoalho pélvico, com consequente aumento de ocorrência de perda de força muscular em mulheres idosas. ${ }^{9,12,13}$

A diminuição da força da MAP é fator etiológico em diversas patologias, como os prolapsos de órgãos, disfunções sexuais, incontinência urinária, dentre outras. ${ }^{14,15}$ A prevalência de prolapsos em mulheres entre 20 e 59 anos é de 30,8\%; cerca de 15 a $20 \%$ das mulheres sexualmente ativas queixam-se de dor pélvica crônica, ${ }^{3}$ estima-se que até $50 \%$ das mulheres sofrerão incontinência urinária em alguma fase da vida e a prevalência de disfunções sexuais é variável. ${ }^{9} \mathrm{~A}$ diminuição da concentração de estrogênio associada à menopausa vem sendo considerada como fator responsável pela alta prevalência de incontinência urinaria em mulheres idosas; ' é provável que aconteçam também repercussões importantes na esfera sexual e na qualidade de vida feminina. $8,12,16$

A fisioterapia dispõe de recursos para promover o ganho de força e reestabelecer as funções da MAP. Destaca-se a cinesioterapia perineal, que reforça o controle esfincteriano uretral e hipertrofia as fibras musculares do assoalho pélvico, ${ }^{17}$ a associação dos exercícios propostos por Kegel em $1950^{9} \mathrm{com}$ os cones vaginais para melhora da consciência e ganho de força, a ativação da musculatura com o auxílio dos equipamentos de eletroestimulação vaginal e eletromiografia - biofeedback, que também facilitam a consciência perineal e a contração muscular. Além destas intervenções, outro meio de proporcionar o fortalecimento desta musculatura é através do método Pilates.

A contrologia, nome original do método, foi desenvolvida ao final da década de 1920, pelo alemão Joseph Hubertus Pilates (1883-1967) e tem como princípios: o movimento do corpo todo, a respiração, o desenvolvimento muscular equilibrado, a concentração, o controle, a centralização da força, a precisão e fluidez do movimento, que juntos procuram desenvolver o corpo de maneira uniforme. ${ }^{18,19}$ O centro de força do corpo humano ou Powerhouse, definido por Joseph $\mathrm{H}$. Pilates, como o local onde se originam todos os movimentos e é composto pelos músculos da parede abdominal anterior, parede abdominal posterior, extensores e flexores do quadril, e assoalho pélvico. ${ }^{19,20} \mathrm{~A}$ ativação do Powerhouse consiste numa contração dos músculos abdominais inferiores somados à co-ativação do assoalho pélvico, aplicando dentre 20 a $30 \%$ da contração voluntária dos músculos envolvidos, e provoca um aumento da pressão intra-abdominal, mas uma leve elevação do AP, capaz de prevenir a Incontinência Urinária de Esforço (IUE). ${ }^{21,22}$

Estudos buscam avaliar os benefícios deste método, porém há poucas referências científicas sobre estes efeitos especificamente no público feminino na fase de transição do período reprodutivo para menopausa. O objetivo desta pesquisa foi avaliar a influência do método Pilates na força de contração da MAP e na qualidade de vida de mulheres no período do climatério.

\section{MÉTODO}

A presente pesquisa, semi experimental do tipo séries de tempo, consistiu de estudo de caso, aprovada pelo Comitê de Ética e Pesquisa do Instituto Superior e Centro Educacional Luterano Bom Jesus/lelusc, através do parecer número $658046 / 2014$, foi desenvolvido no ambulatório de disfunções músculo-esqueléticas do curso de Fisioterapia da Associação Catarinense de Ensino (ACE), em Joinville/SC.

Os critérios de inclusão abrangeram mulheres na faixa etária entre 40 e 55 anos, no período do climatério, que tiveram pelo menos uma gestação a termo, excluindo aquelas que já praticavam o método Pilates 
ou que apresentassem infecções genito-urinárias, gestantes, nulíparas, histerectomizadas ou que fizeram perineoplastia. Foram selecionadas 02 participantes que obedeceram ao perfil da amostra, pacientes 1 e 2 , com 50 e 48 anos respectivamente.

Após a assinatura do Termo de Consentimento Livre e Esclarecido - Resolução 466/2012 - CNS/CONEP, as pacientes foram submetidas a uma pré-avaliação contendo: anamnese, Questionário de Hauser, ${ }^{23}$ Avaliação Funcional do Assoalho Pélvico - AFA, ${ }^{24}$ teste dos Cones Vaginais - TCV, ${ }^{25}$ teste de força muscular específico para fibras de contração rápidas e lentas, com o perineômetro de pressão modelo Perina da marca Quark $^{\circledR}$ e Questionário de Saúde da Mulher - WHQ. ${ }^{26}$

O questionário proposto por Hauser, exposto na tabela 1, é constituído por uma tabela de sinais e sintomas, para a obtenção do índice, a paciente deve dar notas de 0 a 10 para cada um dos sintomas listados, sendo $0=$ ausência do sintoma e 10 = sintoma muito exacerbado. Ao final, somam-se todas as notas e divide-se por 10. Considera-se elevado quando atinge a nota $7 .^{23}$ Esta avaliação foi realizada na pré-avaliação para verificação da presença dos sintomas climatéricos, pelo índice de Hauser: fenômenos vasomotores, palpitação cardíaca, insônia, depressão, irritabilidade, queixas urinárias, concentração e memória, diminuição da libido e secura vaginal.

A AFA consiste na visualização da genitália externa, onde a paciente permanece posicionada na postura ginecológica desnuda da cintura para baixo, verificando-se a ausência ou presença de contração voluntária visível da MAP, após comando verbal. Em seguida é realizada a percepção tatilpalpatória pelo teste digital, onde o avaliador introduz o dedo indicador e o médio no introito vaginal e pede para que a paciente realize contrações da MAP. ${ }^{25}$ Para graduar a força, utilizou-se a classificação funcional do AP de Ortiz: ${ }^{24}$ grau (0) - sem função perineal objetiva, nem mesmo à palpação; grau (1) - função perineal objetiva ausente, contração reconhecível somente à palpação; grau (2) função perineal objetiva débil, contração reconhecível à palpação; grau (3) - função perineal objetiva presente e resistência opositora não mantida mais do que 5 segundos à palpação; grau (4) - função perineal objetiva presente e resistência opositora mantida mais do que cinco segundos à palpação.

Os cones vaginais são dispositivos de tamanhos iguais e pesos diferentes, que variam de $25 \mathrm{~g}$ a $65 \mathrm{~g}$. A Avaliação Funcional do AP realizada com estes dispositivos, inicia-se com a paciente em posição ginecológica, introduz-se na vagina o dispositivo mais leve, deixando-se o fio de nylon para fora e pede-se para a paciente vestir uma calcinha e caminhar durante um minuto, agachar/ levantar e tossir quatro vezes. Caso o cone permaneça no canal vaginal, ele é removido e insere-se o próximo de maior peso e assim sucessivamente, até que algum deles se exteriorize durante os esforços. ${ }^{25} \mathrm{~A}$ classificação do grau de força do assoalho pélvico fez-se de acordo com o peso do cone sustentado no momento da avaliação: Índice TCV: (0) - o peso $\mathrm{n}^{\circ} 1(25 \mathrm{~g})$ cai; (1) - o peso $n^{\circ} 1$ é mantido e o $n^{\circ} 2(35 g)$ cai; (2) - o peso $n^{\circ} 2$ é mantido e o $n^{\circ} 3(45 \mathrm{~g})$ cai; (3) - o peso $n^{\circ} 3$ é mantido e o $n^{\circ} 4(55 \mathrm{~g})$ cai; $(4)$ - o peso $n^{\circ} 4$ é mantido e o $n^{\circ} 5(65 \mathrm{~g})$ cai; (5) - o peso $n^{\circ} 5$ é mantido.
O perineômetro é um aparelho de biofeedback, constituído por dispositivo pneumático de resistência, que afere a contração muscular. O dispositivo é inserido na vagina e conforme a paciente contraí os músculos perineais, um medidor reflete a alteração da pressão exercida pela contração e fornece informações numéricas. Esse instrumento avalia o pico máximo de contração muscular do assoalho pélvico, visualizando no visor do aparelho em $\mathrm{cmH} 2 \mathrm{O}$, que varia de 0 a 256 cmH2O; e com auxílio do cronômetro digital deve-se medir o tempo máximo de contração com esse método. ${ }^{9,27}$ Foram avaliadas as fibras rápidas em contrações de 01 segundo e as fibras lentas em contrações de 05 segundos, seguidas do mesmo tempo de relaxamento, sendo realizadas 05 medições para cada tipo de fibra e o resultado obtido pelo comparativo das médias do antes e depois do tratamento.

A qualidade de vida avaliada através do Questionário de Saúde da Mulher (Women's Health Questionnaire - WHQ), é um instrumento previamente validado para a língua portuguesa, sendo composto por 36 itens agrupados em nove domínios: sintomas somáticos, humor deprimido, dificuldades cognitivas, ansiedade, satisfação sexual, sintomas vasomotores, distúrbios do sono, atração e sintomas menstruais. Quanto maior o escore obtido, pior a qualidade de vida. ${ }^{26}$

O tratamento consistiu em 10 sessões, com frequência de 03 vezes por semana, com duração de 50 minutos cada, iniciando pelo aquecimento da onda respiratória e ativação do Powerhouse, seguidas pelos exercícios do método Pilates: Hundred, Single leg circle, Single leg stretch, Spine stretch, Saw, Double Leg Ki$c k$, Shoulder Bridge, Side kick, Push-up; as repetições foram baseadas no método original. ${ }^{19}$ Os exercícios determinados englobavam mobilização, fortalecimento e alongamento, com ênfase no alinhamento e estabilização lombo-pélvica.

Ao final do tratamento, foi realizada a reavaliação das pacientes e os dados foram analisados através de tabelas do Microsoft Excel, com cálculo da média e desvio-padrão, e comparativo entre o pré e pós-tratamento.

\section{RESULTADOS}

As pacientes 1 e 2 obtiveram no índice de Hauser notas de 6,9 e 6,2 respectivamente, demonstrando a presença dos sintomas climatéricos. Ambas com histórico de duas gestações a termo e parto via cesárea, com atividade sexual presente, e não apresentavam sintomas de perda urinária. Em relação à prática de atividades físicas, a paciente 1 realizava caminhadas com frequência de 4 vezes na semana, enquanto a paciente 2 era sedentária.

Nos testes AFA e TCV as participantes apresentaram grau 5 de força na pré-avaliação, permanecendo inalterados os graus na reavaliação. Notou-se contração consciente em ambas na avaliação inicial, porém com uso de músculos abdominais, o que não é o ideal. Já, ao final do tratamento a paciente 1 apresentou a contração isolada da MAP.

A força muscular do assoalho pélvico, verificada através da perineometria, apresentou melhora relevante. No teste de contração de fibras rápidas, a paciente 1 


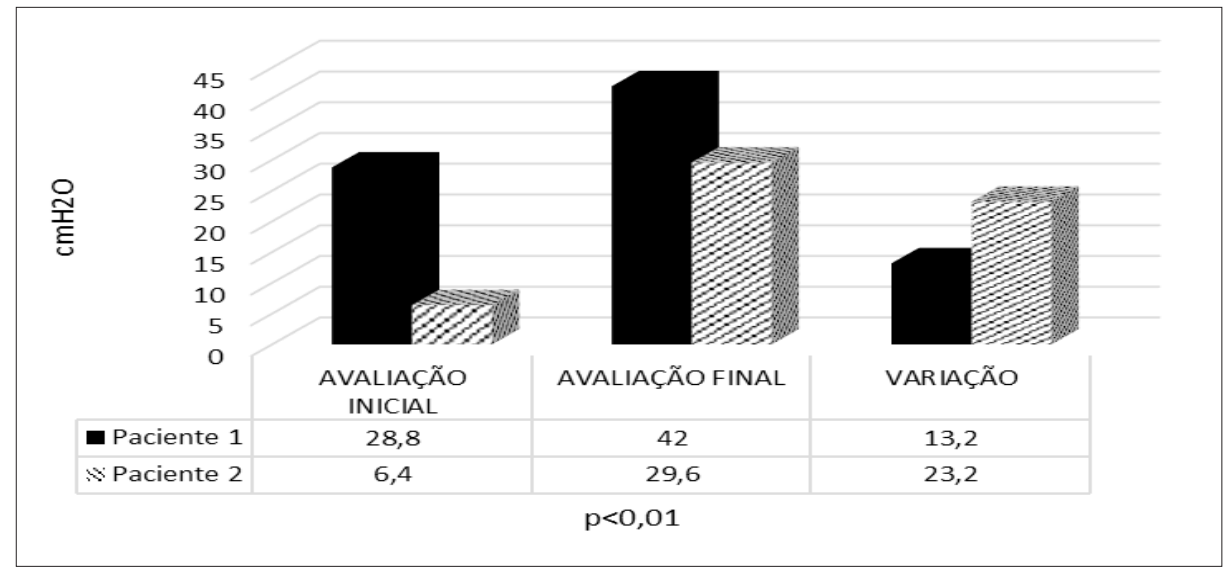

Figura 1 - Avaliação da força muscular, Fibras rápidas, Perinômetro.

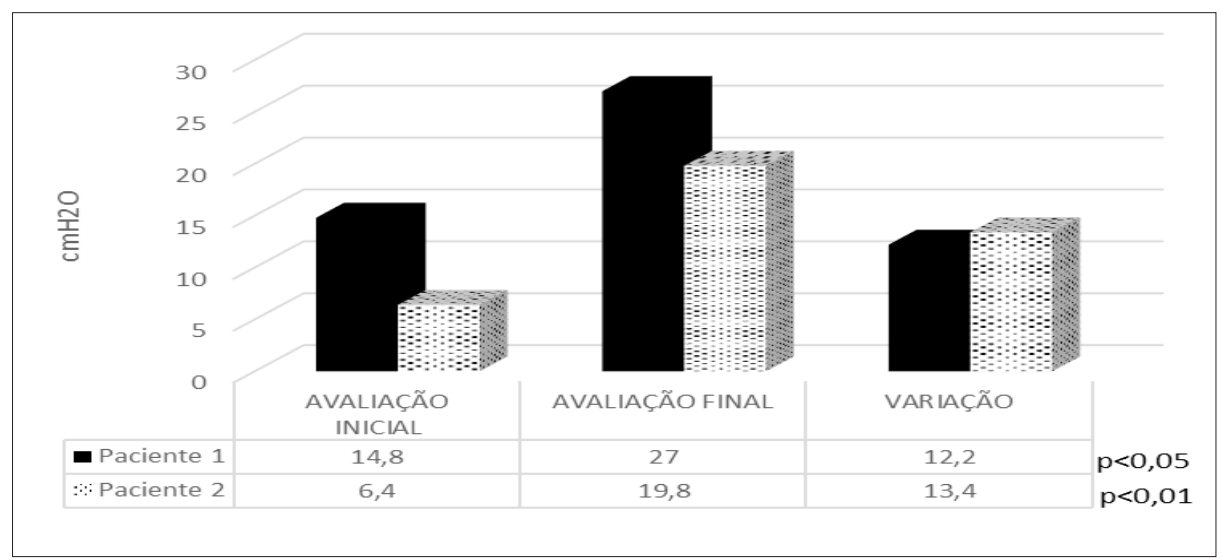

Figura 2 - Avaliação da força muscular, Fibras lentas, Perinômetro.

obteve ganho de força de $45,83 \%(p<0,01)$ e a paciente 2 aumentou em $362,50 \%$. Os resultados em $\mathrm{cmH}_{2} \mathrm{O}$ podem ser observados no gráfico 1 .

$\mathrm{Na}$ avaliação da contração de fibras lentas o incremento de força foi de $82,43 \%(p<0,05)$ e $209,38 \%$ $(p<0,01)$, para as pacientes 1 e 2 , respectivamente, observadas no gráfico 2 .

$\mathrm{Na}$ avaliação da percepção da qualidade de vida pelo WHQ, a paciente 1 apresentou melhora nas dimensões: sintomas somáticos $(14,29 \%)$, sintomas vasomotores $(12,5 \%)$, ansiedade/temores $(12,5 \%)$, problemas de sono $(25 \%)$ e sintomas menstruais $(12,5 \%)$. A paciente 2 apresentou melhora somente nas dimensões: depressão $(10,72 \%)$, sintomas somáticos $(3,58 \%)$, sintomas menstruais $(12,5 \%)$, atratividade $(8,34 \%)$.

\section{DISCUS5ÃO}

O propósito do estudo foi investigar a influência da execução do método Pilates sob a musculatura do assoalho pélvico de mulheres que estão sob o efeito do hipoestrogenismo. Observou-se que no AFA e TCV, testes considerados eficazes, de fácil aplicabilidade e de baixo custo pela literatura, ${ }^{24}$ não havia déficit de força no pré-teste, mantendo os mesmos graus de força pós-tratamento.

Em contrapartida, na análise da MAP através do instrumento perineômetro, apontado como padrão-ouro para a avaliação do $\mathrm{AP}^{25}$ a presente pesquisa apontou a evolução da força muscular dos sujeitos. A musculatura do Powerhouse, trabalhada durante a execução dos movimentos do Pilates, exige a contração de músculos abdominais, estabilizadores da coluna vertebral, iliopsoas, músculos da região lombossacral e também do AP. ${ }^{29}$ A solicitação para que a praticante recrute estes músculos, durante os movimentos, é característico do método, que resulta em um equilibrado desenvolvimento muscular, propicia a estabilidade, aumenta a força postural dinâmica e assegura o funcionamento apropriado da cadeia cinética por inteiro, resultando na estabilização central, importante para o equilíbrio de carga adequado dentro da coluna vertebral e pélvis. ${ }^{29}$ Apesar da contração da MAP solicitada pelo método Pilates ser realizada em conjunto com os músculos abdominais e outros grupos musculares, esta acontece no momento da expiração, em que a pressão intra-abdominal é reduzida e permite a elevação do AP, garantindo sua ativação.

Os resultados foram positivos para ambas as pacientes, e mais significativos na paciente 2 , que era sedentária, corroborando com o estudo ${ }^{30}$ que relacionaram que a menor incidência de IU, associada à perda de força da MAP, pode ser atribuída aos benefícios da atividade física moderada, ao mecanismo de continência, musculatura que foi exigida no tratamento proposto.

Efeitos semelhantes aos da presente pesquisa em relação à força e à pressão de contração, foram encontrados em um estudo que comparou a função do AP 
em mulheres continentes e incontinentes e foi constatada uma resposta aumentada no grupo de pacientes continentes, o que segundo os autores demonstra a importância de se trabalhar preventivamente. ${ }^{31}$

Por fim, outra hipótese foi verificar os efeitos do ganho de força da MAP sobre a QV da amostra. Apesar dos ganhos em relação à força da MAP, o seu reflexo sobre a autopercepção da QV das mulheres foi discreto, porém positivo em algumas dimensões do WHQ, como sintomas somáticos, sintomas vasomotores, ansiedade e temores, problemas de sono, sintomas menstruais, depressão e atratividade. $O$ treinamento da MAP, quando executado regularmente, proporciona a melhora da função muscular do assoalho pélvico, desta forma, acredita-se que a melhora da funcionalidade possa estar diretamente associada à diminuição do número de perdas urinárias e, consequentemente, com a melhora da QV dessas mulheres. ${ }^{32}$ Tais resultados podem ser justificados pela ausência de distúrbios prévios da MAP na amostra, e por outros fatores implicados na autoavaliação da QV.

\section{CONSIDERACÕES FINAIS}

A respiração é um dos princípios fundamentais do método Pilates e atrelada à correta ativação do Powerhouse, exige a contração de diversos músculos, incluindo a MAP. Esta contração ocorre durante todas as expirações realizadas na execução dos exercícios e isto é capaz de promover um ganho de força expressivo nas mulheres que estão no período do climatério, conforme apontam os resultados desta pesquisa. Entretanto, a correlação com o aumento da QV não ocorreu na mesma proporção, o que reforça a necessidade de outros estudos, destinados à pacientes com patologias associadas e outras variáveis.

\section{REFERÊNCIAS}

1. Silva APS, Silva JS. A importância dos músculos do assoalho pélvico feminino sob uma visão anatômica. Fisioter Bras 2003;4(3):205-10.

2. Oliveira C, Lopes MAB. Efeitos da Cinesioterapia no assoalho pélvico durante o ciclo gravídico-puerperal [dissertação]. São Paulo (SP): Universidade de São Paulo; 2006.

3. Grosse D, Sengler J. Reeducação perineal: concepção, realização e transcrição em prática liberal e hospitalar. São Paulo: Manole; 2002.

4. Gosling JA, Dixon JS, Critchley HOD, Thompson SA. A comparative study of the human external sphincter and periurethral levator ani muscles. Br J Urol 1981;53(1):35-41.

5. Ribeiro, Mara Cláudia. Impacto do hipoestrogenismo sobre a força muscular do assoalho pélvico em mulheres pósmenopáusicas [Dissertação]. Brasília (DF): Universidade Católica de Brasília: 2010.

6. Lustosa $A B$, Girão MJBC, Sartori MGF, Baracat EC, Lima GR. Citologia hormonal do trato urinário baixo e da vagina de mulheres na pós menopausa, antes e durante estrogenioterapia oral e transdérmica. Rev Bras Ginecol Obstet 2002;24(9):573577. doi: 10.1590/S0100-72032002000900002

7. Rechberger T, Skorupski P. The controversies regarding the role of estrogens in urogynecology. Folia histochemica et cytobiologica 2007;45(1):17-21.

8. De Lorenzi DRS, Danelon C, Saciloto B, Padilha JR I. Fatores indicadores da sintomatologia climatérica. Rev Bras Ginecol Obstet 2005;27(1):12-19. doi: 10.1590/S0100-72032005000100004

9. Baracho E. Fisioterapia aplicada à obstetrícia, urologia e aspectos de mastologia. 4. ed. Rio de Janeiro: Guanabara Koogan; 2007.

10. Lopes MEL, Morais MAC. Identidade da mulher em fase de climatério: aspectos histórico-culturais e educacionais. $4^{\circ}$ Congresso Brasileiro de História da Educação. Anais; 2006. Disponível em: http://www.sbhe.org.br/novo/congressos/ cbhe4/individuais-e-co-autorais-ixo04.htm.

11. Silva Filho EA, Costa AM. Avaliação da qualidade de vida de mulheres no climatério atendidas em hospital-escola na cidade do Recife, Brasil. Rev Bras Ginecol Obstet 2008; 30(3):113-20. doi: 10.1590/S0100-72032008005000001

12. Goldstein $S R$, Neven $P, Z$ hou $L$, Taylor $Y L$, Ciaccia AV, Plouffe LJ. Raloxifene effect on frequency of surgery for pelvic floor relaxation. Obstetrics \& Gynecology 2001;98(1):91-96.

13. Rovner ES, Wein AJ. Treatment options for stress urinary incontinence. Reviews in Urology 2004;6(3):29-47.

14. Bernardes ON, Péres RF, Souza LBLE, Souza LO. Métodos de tratamentos utilizados na incontinência urinária de esforço genuína: um estudo comparativo entre cinesioterapia e eletroestimulação endovaginal. Rev Bras Ginecol Obstet 2000;22(1):49-54. doi: 10.1590/S010072032000000100009

15. Pivetta HMF, Braz MM, Real AA, Nascimento JR, Cabeleira MEP, Veye APZ. Disfunções do assoalho pélvico em pacientes submetidas à histerectomia: um estudo de revisão. Cinergis 2014;15(1):48-52.

16. Freitas, E. V.; Py, L.; Cançado, F. A. X.; Doll, J.; Gorzoni, M. L. Tratado de Gerontologia. 2ed. Rio de Janeiro: Guanabara Koogan 2006:766-775.

17. Luz SCT da, Virtuoso JF, Puhlmann TPM, Coan MV, Krüger AP, Honório GJS. Educação perineal progressiva EPP: em busca da continênca urinária. São Paulo: Biblioteca24horas; 2011.

18. Aparício E, Pérez J. O Autêntico método pilates: a arte do controle. São Paulo: Planeta do Brasil; 2005.

19. Pilates JH, Miller WR. Return to life through contrology. Miami: Pilates Method Alliance, Inc; 2005.

20. Muscolino JE, Cipriani S. Pilates ant the 'powerhouse'. I. Journal of Bodywork and Movement Therapies 2004;8:15-24.

21. Queiroz BC, Cagliari MF, Amorim CF, Sacco IC. Muscle activation during four pilates core stability exercises in quadruped position. Arch Phys Med Rehabil 2010;91(1):8692. doi: 10.1016/j.apmr.2009.09.016

22. Sapsford RR, Hodges PW. Contraction of the pelvic floor muscles during abdominal maneuvers. Arch Phys Med and Rehabil 2001;82(8):1081-88.

23. Hauser GA, Huber IC, Keller PJ, Lauritzen C, Schneider HP. Evaluation of climacteric symptoms (Menopause Rating Scale). Zentralbl Gynakol 1994;116:16-23.

24. Ortiz OC, Coya NF, Ibanez G. Evaluación funcional del piso pelviano femenino (clasificación funcional). Soc Latinoam Uroginecol Cir Vaginal. 1996;1:5-9.

25. Nascimento SM. Avaliação fisioterapêutica da força muscular do assoalho pélvico na mulher com incontinência urinária de esforço após cirurgia de Wertheim-Meigs: revisão de literatura. Rev Bras Cancerol 2009;55(2):157-63.

26. Silva Filho CR, Baracat EC, Conterno LO, Haidar MA, Marcos Bosi Ferraz, MB. Sintomas climatéricos e qualidade de vida: validação do questionário da saúde da mulher. Rev 
Saúde Pública 2005;39(3):333-39. doi: 10.1590/S0034 89102005000300002

27. Stephenson RG, O Connor LJ. Fisioterapia aplicada à Ginecologia e Obstetrícia. 2. ed. São Paulo: Manole, 2004.

28. Bianco G, Braz MM. Efeitos do exercício do assoalho pélvico na sexualidade feminina. (artigo original) Tubarão (SC): UNISUL; 2004.

29. Marés G, de Oliveira KB, Piazza MC, Preis C, Bertassoni L Neto. A importância da estabilização central no método Pilates: uma revisão sistemática. Fisioter Mov 2012;25(2):445-51. doi: 10.1590/S0103-51502012000200022

30. Virtuoso JF, Mazo GZ, Menezes EC. Prevalência, tipologia e sintomas de gravidade da incontinência urinária em mulheres idosas segundo a prática de atividade física.
Fisioter Mov 2012;25(3):571-82. doi: 10.1590/S010351502012000300013

31. Souza CEC, Lima RM, Bezerra LMA, Pereira RW, Moura TK, Oliveira RJ. Estudo comparativo da função do assoalho pélvico em mulheres continentes e incontinentes na pós menopausa. Rev Bras Fisioter 2009;13(6):535-41. doi: 10.1590/S1413-35552009005000060

32. Fitz FF, Costa TF, Yamamoto DM, Resende APM, Stüpp L, Sartori MGF, Girão JBC, Castro, RA. Impacto do treinamento dos músculos do assoalho pélvico na qualidade de vida em mulheres com incontinência urinária. Rev Assoc Med Bras 2012;58(2):155-59. doi: 10.1590/S010442302012000200010 